\title{
Modeling of VIPMOS Hot Electron Gate Currents
}

\author{
G.J. Hemink, R.C.M. Wijburg, P.B.M. Wolbert, and H. Wallinga
}

MESA Research Institute, University of Twente, Faculty of Electrical Engineering, P.O. Box 217, 7500 AE Enschede, The Netherlands.

\begin{abstract}
A buried injector, which is biased by means of punch-through, can be used in substrate hot electron injection EEPROM devices [1]. In order to optimize this device an empirical expression for the injection probability as a function of the effective barrier height and the average electron energy is proposed and verified by measurements on a variety of devices.
\end{abstract}

\section{Introduction}

Various models are used to compute the effect of hot electron injection. A well known model is the Lucky Electron Model (LEM) [2]. As will be shown this model is not able to predict the gate current with sufficient accuracy. Other models are derived from a solution of the Boltzmann transport equation [3] or from time-consuming MonteCarlo simulations [4]. In [5] it is shown that the Richardson equation, that only requires the average electron energy as a parameter, overestimates the hot electron gate currents for several orders of magnitude. This is because the energy distribution function is nonMaxwellian for high average electron energies [3-5]. An empirical model which only requires the average electron energy, but does take into account the effect of a nonMaxwellian distribution function, will be presented.

\section{The VIPMOS structure}

The VIPMOS (Vertical Injection Punch-through based MOS) structure is given in figure 1. During hot electron injection the injector is grounded and the gate is connected to a sufficiently high voltage. Increasing the voltage on both the source and the drain will extend the depletion layer under the gate into the direction of the injector. At a certain voltage punch-through occurs and electrons are injected into the depletion layer under the gate. The electrons are accelerated in the high electric field and some of them gain sufficient energy to

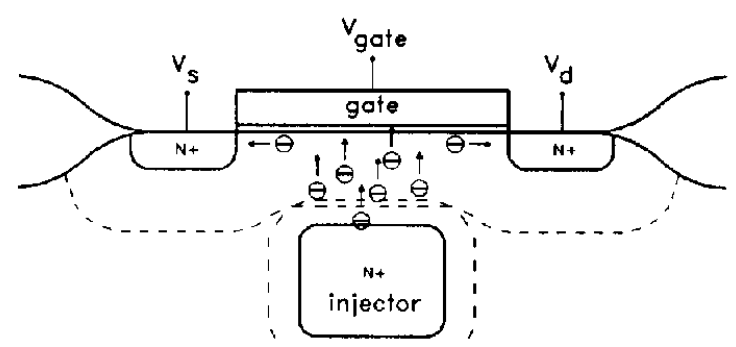

Figure 1. The VIPMOS structure. 
surmount the $\mathrm{Si} / \mathrm{SiO}_{2}$ potential barrier. The injection probability is defined as $\mathrm{I}_{\text {gatc }} / \mathrm{I}_{\mathrm{inj}}$ and depends on the doping profile between the buried injector and the $\mathrm{Si} / \mathrm{SiO}{ }_{2}$ interface [1].

\section{Simulations and measurements}

In figure 2, the significantly different doping profiles of four devices are shown. These doping profiles have been simulated with SUPREM3. All the device- and hydrodynamic simulations have been carried out with TRENDY [6]. Figure 3 shows the simulated and measured punch-through current density as a function of the programming voltage. As can be concluded from these results, the devices 1,2 and 3 can be used in 5 Volt only applications. Device 1 can even be used with a programming voltage of 3.3 Volt. If the potential in the injector structure is known then the Lucky Electron Model (LEM) [2] can be applied. The injection probability is then given by:

$P_{\text {inj }}=A \exp (-d / \lambda)$

where $\mathbf{A}$ is a fitting constant, $\lambda$ is the mean free path parameter and $d$ is the minimum free path an electron has to travel in order to acquire an energy equal to the effective $\mathrm{Si} / \mathrm{SiO}_{2}$ barrier height. In order to compute $\mathrm{d}$, the following expression for the effective barrier height is used [2]:

$\phi_{\mathrm{b}}=3.1-\beta \mathrm{E}_{\mathrm{ox}}^{1 / 2}-\alpha \mathrm{E}_{\mathrm{ox}}^{2 / 3} \mathrm{eV}$

with $\mathrm{E}_{\mathrm{ox}}$ the electrical field in the gate oxide, $\alpha$ and $\beta$ are constants. The parameter $\beta$ is incorporated to model the effect of image force barrier lowering and has a value of $2.59 \cdot 10^{-4} \mathrm{e}(\mathrm{V} \cdot \mathrm{cm})^{1 / 2}$. The value of parameter $\alpha$ is $1.0 \cdot 10^{-5} \mathrm{e}\left(\mathrm{cm}^{2} \cdot \mathrm{V}\right)^{1 / 3}$, and is used to account for the tunneling probability [2].

The simulated potential under the punchthrough condition is given in figure 4. Using these results, it is possible to compute the injection probabilities for the four devices. Figure 5A shows the injection probabilities according to the LEM together with the measured injection probabilities. The parameters $A$ and $\lambda$ have been fitted to the measured data. The best fit is obtained with $A=0.149$ and $\lambda=11.9 \mathrm{~nm}$. This value for $\lambda$ is

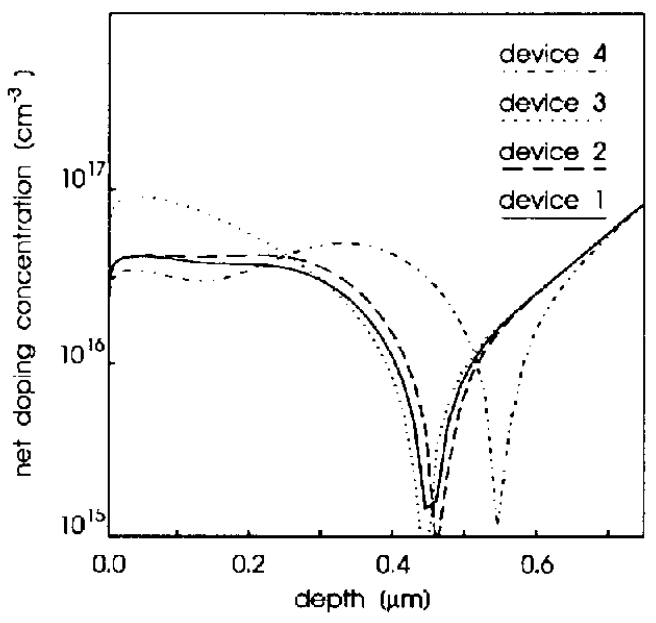

Figure 2. Injector doping profile of four devices. The $\mathrm{Si} / \mathrm{SiO}_{2}$ interface is located at 0 .

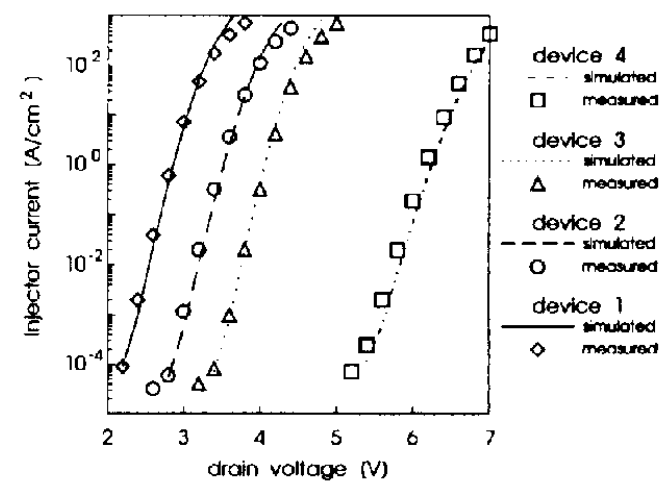

Figure 3. Injection current density as a function of the programming voltage. 
in reasonable agreement with the value of $9.1 \mathrm{~nm}$ that is found by Ning [2] at a lattice temperature of $300 \mathrm{~K}$. Figure $5 \mathrm{~A}$ also shows that the LEM fails to predict the injection probability of device 4 correctly. For a more exact computation of the injection probability, knowledge about the electron energy distribution function $f(\varepsilon)$ is required. In general this distribution is non-Maxwellian and can only be obtained by means of time-consuming Monte-Carlo simulations. In order to overcome this problem the following empirical relation for the injection probability as a function of the effective barrier height $\phi_{b}$ and the average electron temperature $T_{c}$ at the $\mathrm{Si} / \mathrm{SiO}_{2}$ interface is proposed:

$P_{\text {inj }}=A \exp \left(-B\left(\frac{\phi_{b}}{k T_{e}}\right)^{n}\right)$

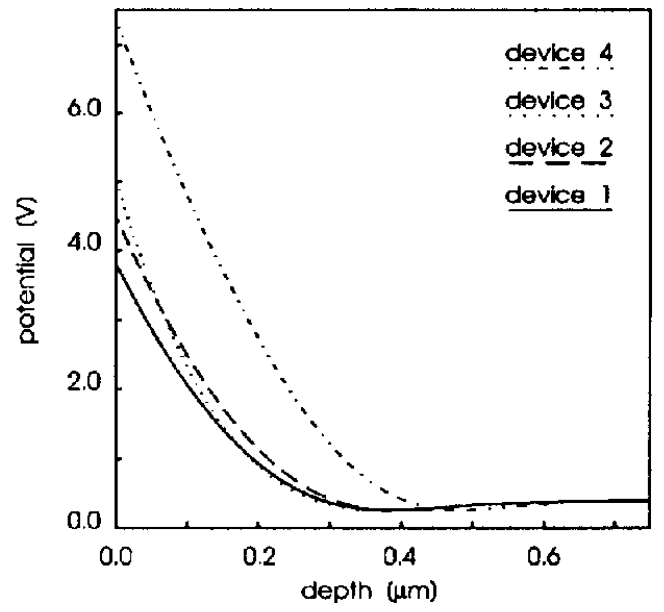

Figure 4. Potential in the injector structure during injection with a current density of $100 \mathrm{~A} / \mathrm{cm}^{2}$

in which $A, B$ and $n$ are fitting constants and $T_{e}$ is the average electron temperature. When the average electron energy $\varepsilon$ is known then $\mathrm{T}_{\mathrm{e}}$ can be approximated by using:

$\varepsilon=\frac{3}{2} \mathrm{kT}_{\mathrm{e}}$

The parameters $B$ and $n$ are used to describe the effect of a non-Maxwellian energy distribution function. The average electron energy is computed by solving the energy balance equations [6]. The energy relaxation time that is used in these simulations is $550 \mathrm{fs}$. The results of these computations are shown in figure 6 . Using these results, the

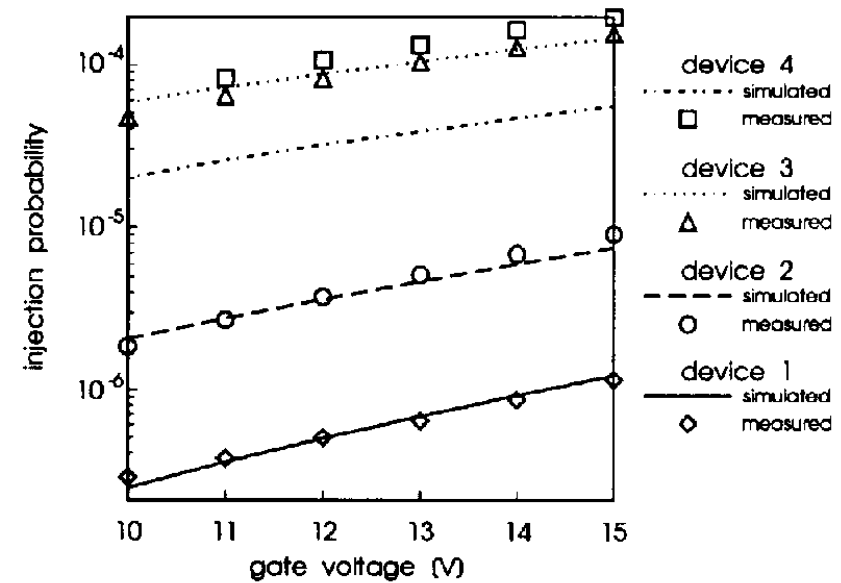

(A)

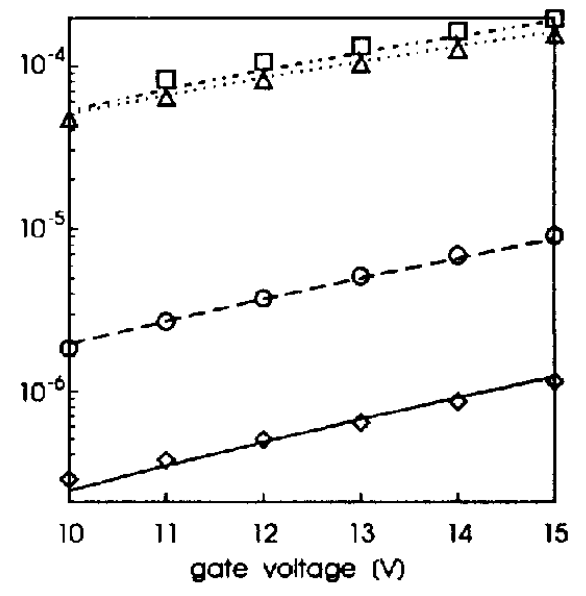

(B)

Figure 5. Measured and simulated injection probabilities according to (A) the Lucky Electron Model and (B) according to equation (3). 
injection probability can be computed by using equation (3). For the computation of the effective barrier height, the expression in equation (2) was used. The parameters A,B and $n$ have been fitted with a parameter extraction program. The values of these parameters are: $\mathrm{A}=4.97 ; \mathrm{B}=3.16$; and $\mathrm{n}=1.10$ Figure $5 B$ shows the measured and simulated injection probabilities using equation (3). A very good fit is obtained for all devices.

\section{Discussion}

Figure 5A shows that the LEM prediction of the injection probability of device 4 is incorrect. This is because the LEM does not take into account the average energy of the electrons at the distance d. The minimum

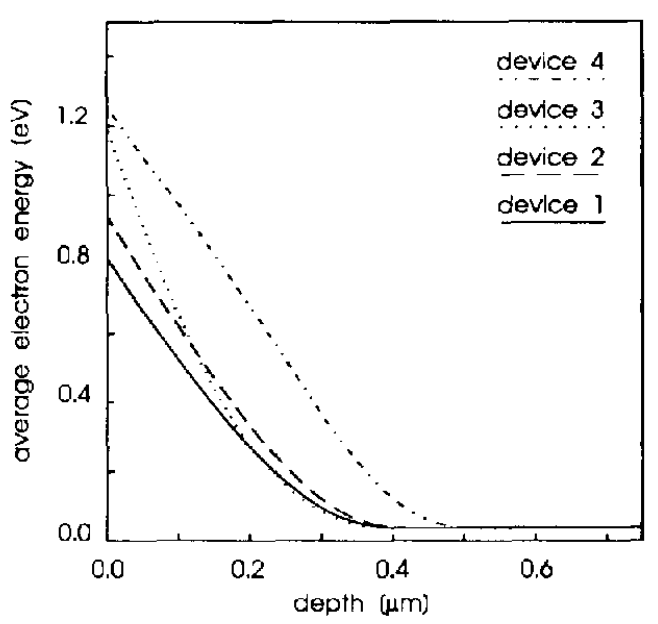

Figure 6. Average electron energy during injection with a current density of $100 \mathrm{~A} / \mathrm{cm}^{2}$. free path $\mathrm{d}$ is $88 \mathrm{~nm}$ for device 3 and $100 \mathrm{~nm}$ for device 4 (both at a gate voltage of $12 \mathrm{~V}$ ). Using equation (1) results in a higher injection probability for device 3 in comparison with device 4 . Figure 6 shows that there is a significant difference of about $0.25 \mathrm{eV}$ in the average electron energy for the devices 3 and 4 at their corresponding distances $d$. Therefore the actual injection probability of device 4 is higher than predicted by equation (1).

\section{Conclusions}

An empirical model is presented that can be used to compute VIPMOS hot electron gate currents more accurately. The model only requires the average electron energy at the $\mathrm{Si} / \mathrm{SiO}_{2}$ interface, which can be easily simulated by solving the energy balance equations simultaneously with the usual device equations. This model will be used to optimize the VIPMOS device for programming voltages in the 3 Volt range.

\section{Acknowledgements}

This work was supported by the Foundation for Fundamental Research on Matter (FOM), the Netherlands Technology Foundation (STW) and the Philips Research Labs.

\section{References}

[1] R.C.M. Wijburg et. al., IEEE Trans. Electron Devices, vol. 38, pp. 111-120, 1991.

[2] T.H. Ning, J. Solid-State Electron., Vol. 21, pp. 273-282, 1978.

[3] N. Goldsman et. al., Solid-St. Electron., Vol. 31, No. 6, pp. 1089-1092, 1988.

[4] B. Ricco et . al., IEDM Tech. Dig., pp. 559-562, 1986.

[5] S.-L. Wang et. al., IEDM Tech. Dig., pp. 447-450, 1990.

[6] P.B.M. Wolbert et. al., Proc. of NASECODE VII, pp. 126-127, 1991. 